\title{
Enhance Location Based Power Aware Routing Protocol in Ad-Hoc Network
}

\author{
Lal Pratap Verma ${ }^{l}$ \\ Department of Computer Science \& \\ Engineering SRMIET Ambala, \\ Haryana, India
}

\author{
Aniket Mathuriya $^{2}$ \\ Department of Computer Science \& \\ Engineering SRMIET Ambala, \\ Haryana, India
}

\author{
Rajneesh Manwall ${ }^{3}$ \\ Department of Computer Science \& \\ Engineering SRMIET Ambala, \\ Haryana, India
}

ABSTRACT- In wireless ad-hoc networks, there are several characteristics different with wired networks. The differences are changing of network topology, limited resources like bandwidth and energy and so on. In these days as the bandwidth issue becomes more important, it is required to research about the efficient resource allocation methods optimized in wireless network ks. In the paper, we improve LAR (Location-Aided Routing) which is one of the most famous location based routing methods. This kind of technique uses information about the location of mobile node through GPS technique. Our new protocol considers both areas of routing and bandwidth. At first, propose a more efficient routing method which improves the quality of services .Secondly, a bandwidth aware method is proposed to select proper transmission bandwidth node by using a threshold value of bandwidth.

\section{INTRODUCTION}

A key feature of future mobile wireless networks is the ability to adapt and exist even without a fixed infrastructure. An ad hoc network is a collection of possibly mobile devices or nodes that can establish communications, without a fixed infrastructure or central administration. Ad hoc networking is expected to help fulfill the dream of a seamless network architecture and to play an important role in next generation wireless networks and services.

Thus, the task of efficient routing the data packets in terms of QoS and energy consumption and bandwidth becomes very important. Many routing protocols have been proposed for efficient routing. Earlier on-demand routing protocols were based on flooding the routing packets in all directions irrespective of the location of the destination node, result increase bandwidth consumption where as table driven protocol maintains large amount of information as well as they perform large computations in order to select the best node

which results in premature loss of battery life. This bandwidth consumption was reduced by the Location Aided Routing Protocols. These location based protocols uses the Global Positioning System (GPS) to find the direction of propagation of the packets. By finding the direction of propagation we can decrease the bandwidth consumption. In this paper, we are proposing a power aware routing approach which helps to decreasing the routing overhead by utilizing the concept of global location information and provide optimal path en terms of bandwidth. The proposed protocol Enhance Location Based Power Aware Routing (ELBPAR) protocols use location information to minimize the Request Zone to reach the destination node with a certain amount of bandwidth.

\section{RELATED WORK}

There are many location based routing protocol that provide path from source to destination in terms of QOS parameter. These routing protocol uses following parameter.

\section{EXPECTED ZONE}

Consider, source node $S$ wants to send the data packet to destination node D at time $\mathrm{T}$ [8]. Assume that $\mathrm{S}$ knows the location of $\mathrm{D}$ at time Ti. Also it also knows velocity (VD) of $\mathrm{D}$ with which $\mathrm{D}$ is traveling. Maximum distance traveled by $\mathrm{D}$ in any direction can be calculated as:

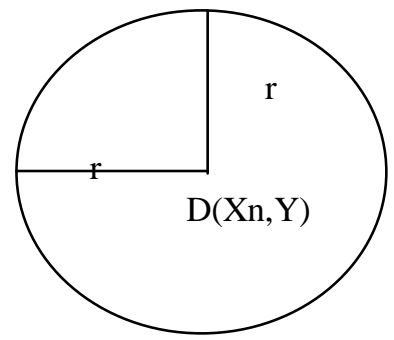

Figure 3.1 Expected Zone

$$
\boldsymbol{r}=V_{D}\left(\boldsymbol{T}-\boldsymbol{T}_{\boldsymbol{i}}\right)
$$

\section{REQUEST ZONE}

Request zone is the area where the request packets are sent or broadcast to find a path from source to destination. In the traditional routing algorithms it is the complete network [8]. For e.g. In AODV, DSR, etc. RREQ packet is broadcasted in all directions to find the optimal path from source to the destination node. LAR tries to minimize the request zone by confining it to the smallest rectangular area containing both sender as well as receiver.

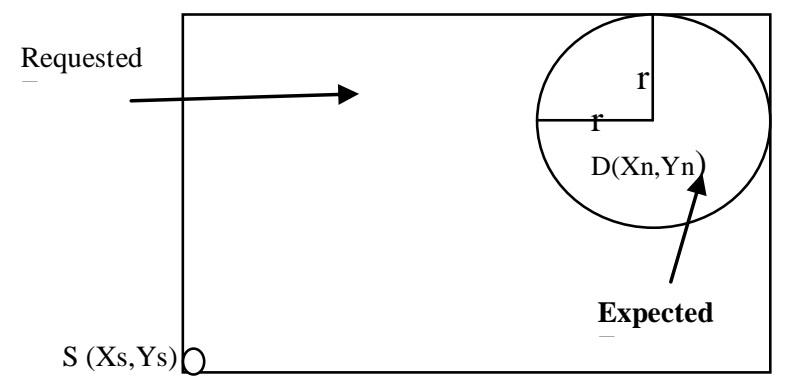

\section{GLOBAL Position SYSTEM}

GPS is a system of satellites [3], ground control stations, and receivers that allows users to determine their position. 
By capturing and storing that position, GPS receivers "digitize" spatial data as they walk, drive, or otherwise traverse the land. Receivers differ in their ability to receive and process GPS signals and users can have a huge affect on accuracy depending on the methods used to collect and process data.

\section{TYPE OF ROUTING PROTOCOL}

$\mathrm{T}$ here are following type of routing protocol those are used by Location Based Routing Protocol.

\section{ENERGY AWARE LOCATION BASED ROUTING PROTOCOL}

Energy aware location based routing technique use location information by using GPS system and additionally takes a grid method. In this routing protocol each node has GPS in Ad-Hoc network divided by virtual grid [10]. This assumption means that each node knows the location of itself and where they are included in the grid network. To maintaining information of neighborhoods, nodes in each grid communicate each other by beacon message which includes ID, geographical location, and energy. From this communication, node which has the largest energy becomes grid header. And header node is changed when the rest of its energy equals to $1 / 2$ of second node. Grid headers communicate each other by longer period than the one of general node. The data sent by each grid header includes information of all nodes in its grid. So grid header can get the information of entire nodes.

\section{IMPROVED LOCATION AIDED ROUTIN}

ILAR (Improved Location Aided Routing) is another location based technique which uses the concept of base line lying in between the source and destination node. Node which is closest to this line of sight will be chosen as the next intermediate node. As the transmitting node check the distance of every neighboring node from base line and find the closest neighbor for further transmission.

3. LOCATION AWARE ROUTING PROTOCOL WITH DYNAMIC ADAPTATION OF REQUEST ZONE

Mobile Ad hoc Networks is an on demand routing protocol which decreases the search area given by LAR[2]. In LAR the search area is the smallest rectangle containing both sender as well as receiver. LARDAR reduces this rectangle to triangular zone, which helps in reducing the routing overheads. But the calculations done to find whether the node lie in the forwarding zone or not increases the overhead on the node. This result in decrease in battery of the node

\section{LOCATION BASED POWER AWARE ROUTING}

LARDAR uses the concept of Triangle zone and the angles $\alpha$ and $\beta$. The protocol LBPAR uses the concept of triangle zone [6]. But instead of using the angular values in route request packet as in LARDAR it using the concept of slopes of line (Figure 3.3) which can be calculated using the following formula

$$
\boldsymbol{m} \boldsymbol{p}=(\boldsymbol{X P}-\boldsymbol{X} \boldsymbol{S}) /(\boldsymbol{Y P}-\boldsymbol{Y S})
$$

The Base objective of LBPAR is to find an optimal path in terms of bandwidth consumption along with the reduction in power loss of a node. Here, the route discovery process starts when source node $\mathrm{S}$ initiates a request to send the data packet to destination node D. $\mathrm{m} 1$ and $\mathrm{m} 2$ are the slopes of the line. $\mathrm{D}(\mathrm{XT}, \mathrm{YT})$ are the points where the tangents drawn from source to the expected zone touches the expected zone. From the

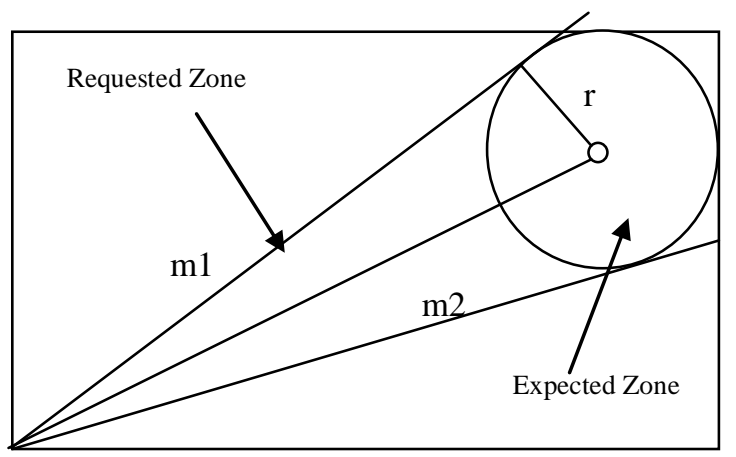

mathematical equations it is clear that $\mathrm{m} 1$ and $\mathrm{m} 2$ will be the maximum and minimum slopes for any line drawn between the source and any point in between these two tangents. These two slopes [5] can be calculated from the following quadratic equation:

$$
m X d-m X s-Y d+Y s=r *\left(1+m^{2}\right)^{1 / 2}
$$

Source node $S$ broadcast a route request packet to find the path. Format of RREQ packet on receiving the RREQ packet node ' $\mathrm{N}$ ' calculates the slope $m_{n}$ using its own location co-ordinates and source location co-ordinates. If its slope lies between the max and min slope i.e. $\left(\mathrm{m} 1 \geq m_{n}\right.$ $\geq \mathrm{m} 2$ ) then node will again broadcast the packet otherwise node drops the packet. This routing protocol is use to routing packets between mobile nodes in an ad hoc network using the Global Positioning System. Unlike routing protocols like LAR, ILAR, LARDAR this protocol considers various parameters like bandwidth requirement and battery life of all the intermediate nodes on a path to destination. Increase in the battery life tends to high probability of routing path. Based on evaluation of LBPAR is proved to be a better protocol as it helps in lowering the bandwidth consumption of the network and also helps in increasing the battery life by decreasing the number and complexity of calculations.

\section{PROBLEM DEFINITION}

Location Based Power Aware Routing (LBPAR) protocols use location information to minimize the Request Zone to reach the destination node. LBPAR will also help in reducing the overheads at each node by decreasing the number of calculations performed at each node, which in turn increased the battery life of node. But it can't provide the guarantee to delivery of packet on existing path due to more demand of bandwidth for sending the packet from source to destination. For example if source A want to send the data to the destination $\mathrm{F}$ by using path A-B-E-F. Every node in this path has his available bandwidth. When data packet is travelling between these source and destination, every node on that path also have some data or RREQ packet or RREP packet to send to other node on this network. Then at that time when A want to send data packet to $\mathrm{F}$, intermediate node $\mathrm{B}$ and $\mathrm{E}$ not have sufficient bandwidth to send data receiving from node $\mathrm{A}$ to $\mathrm{B}$ or $\mathrm{B}$ to 
E . It means that when node B or E are not have sufficient bandwidth to forward the data packet to his neighbor. So these nodes are dropping the data packet.

\section{PROPOSED WORK}

The proposed protocol is an efficient routing scheme that improves the efficiency of location-aided routing (LAR) which improves the quality of services. Proposed scheme is an extended version of Location Based power Aware Routing. In this routing protocol, there are some parameters is use for calculation of expected zone and requested zone are minimum slope, maximum slope, TTL and minimum required bandwidth for communication. Route request packet can be send to the neighbor node based on the minimum, maximum slope and minimum required bandwidth. This protocol has an extra parameter

\section{MINIMUM REQUIRED BANDWIDTH}

Minimum bandwidth is the parameters of packet header that contain minimum require bandwidth for communication between sources to destination.

\section{PHASES OF ELBPAR}

There are three phase in this protocol
I. Route discovery
II. Route Reply
III. Route maintenance

1. Route discovery: $S(X s, Y s)$ is the source node which want to communicate with destination $D(X d, Y d)$ and $1,2,3,4,5,6,7$ are intermediate nodes. Source node $S$ calculate expected zone by using destination coordinate and then calculate the requested zone by using the expected zone. Source node $S$ initiate the RREQ request that packet contain the information like TTL, min_slope, max_slope, min_BW, broadcast id, source address, and destination address. All these information is collected by source node before sending the RREQ packet to his neighbors. When source node neighbors receive the RREQ packet then first of all it check the destination address of RREQ, if destination address is the address of any one of the neighbor node then it consume the packet and send RREP packet to the source node. If destination address is not equal to the neighbor node address then it check the value of TTL, if TTL is less than or equal to zero the node discard the RREQ, else it calculate the slope of from source node. If slope lies between the maximum slope and minimum slope, then it checks whether node bandwidth is greater than or equal to the minimum bandwidth require for communication of source node or not. If node bandwidth is greater than or equal to minimum bandwidth then attach its own address to list of visited node and forward the RREQ packet to its neighbor nodes. If route cannot qualify neither minimum slope and maximum slope nor minimum bandwidth, then node discard the RREQ packet or any one of this parameter then discard the RREQ packet.

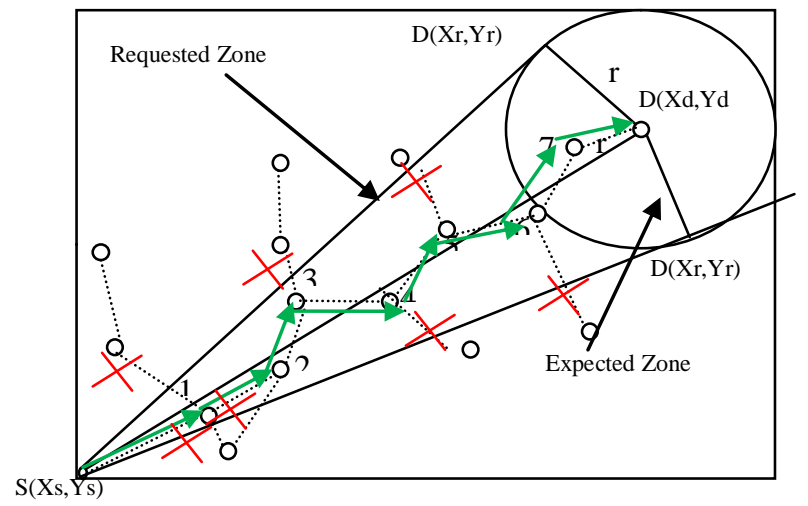

Figure Route Discovery process of ELBPAR

This process is follows by every node which receive the RREQ packet until the RREQ receive the by destination node. Above figure is an example of route discovery process of ELBPAW which find the path between source need $S$ and destination node $D$. Source node $S$ to destination node $\mathrm{D}$ path is $\mathrm{S}-1-2-3-4-5-6-7-D$. Figure

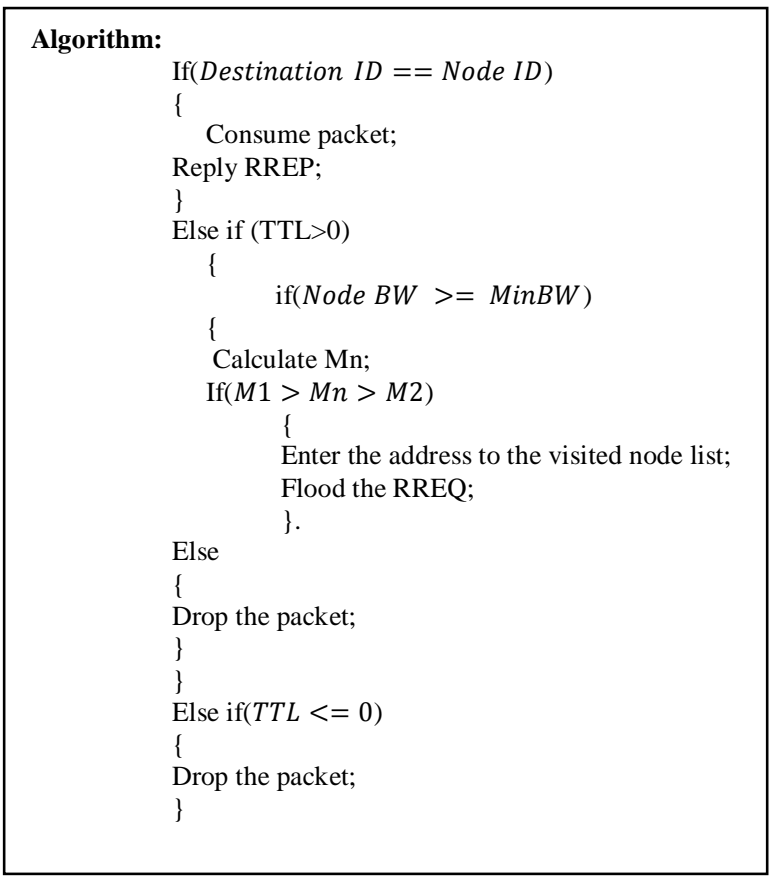

Algorithm for ELBPAR

\section{ROUTE REPLY PHASE}

In route reply phase if destination receives the RREQ packet then it check the packet header destination address. If destination address is the address of node then it check minimum and maximum slope. If slope lei between these two slope then it check bandwidth of that node. If bandwidth is greater than or equal to the minimum bandwidth the destination consume the RREQ packet and sent RREP packet to source node via same path which path is use to receive RREQ packet from source to destination. Otherwise discard the RREQ packet. Figure show the route reply path from destination to source node as D-7-6-5-4-3-2-1-s. 


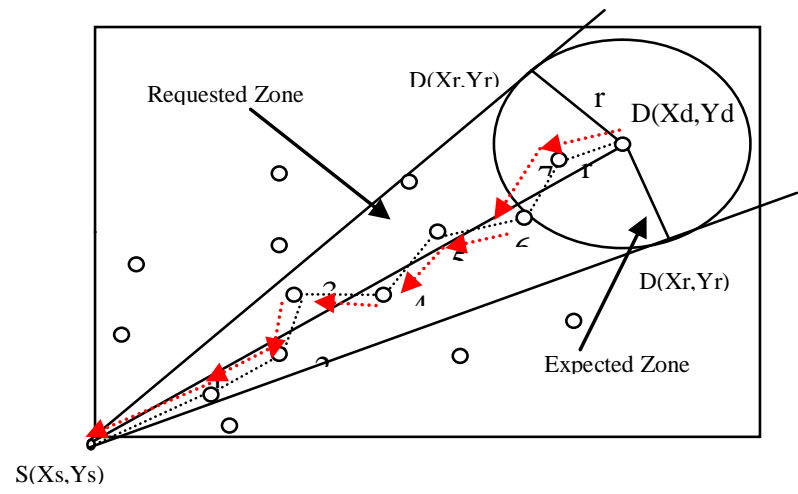

Figure Route Reply Phase of ELBPAR

\section{ROUTE MAINTENANCE}

In Ad-hoc network there is high mobility of nodes, links between nodes are likely to break. Thus, we need to maintain the routing path. For example node 4 in Figure move outside to the reach of its neighbor then route from source to destination is break. In this case route maintenance process is use to maintain the route from source to destination. In this phase when a node does not receive a RREP packet it will break the path. In this case, the node sends a route error (RERR) packet to the source node. When the source node receives the packet, it will reconstruct a new path to the destination node. In Figure node 4 move outside of the reach of its neighbors, so path is brooked. Then again new path is created by source node using node 8 . So the source to destination path after maintenance is $\mathrm{S}-1-2-3-8-5-6-7-\mathrm{d}$.

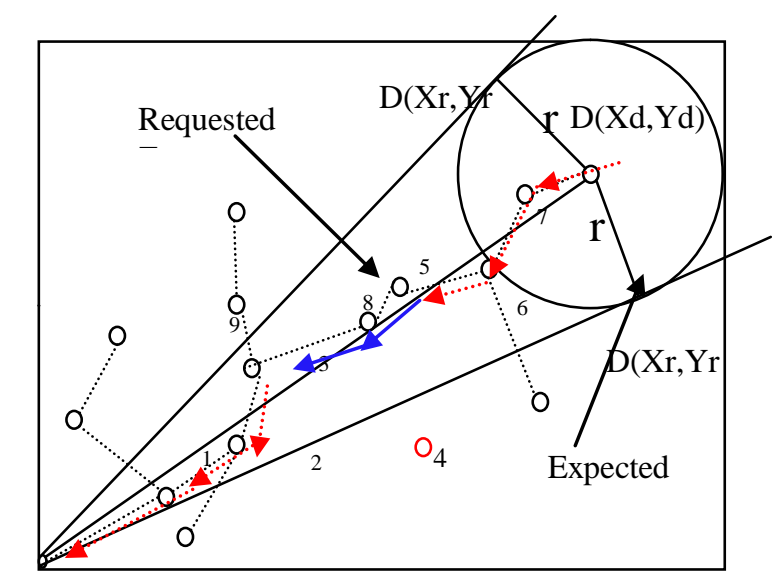

$\mathrm{S}(\mathrm{Xs}, \mathrm{Y}$

Figure Route Maintenance Phase of ELBPAR

\section{CONCLUSION \& FUTURE WORK}

In wireless ad-hoc networks, there are several characteristics different with wired networks. The differences are changing of network topology, limited resources like bandwidth and energy and so on. LAR (Location-Aided Routing) which is one of the most famous locations based routing methods that uses information about the location of mobile node through GPS technique. Our new protocol "Enhance Location Based Power Aware Routing" considers both areas of routing and bandwidth. At first, propose a more efficient routing method which improves the quality of services in terms of routing overhead. Secondly, a bandwidth aware method is proposed to select proper transmission bandwidth of node by using a threshold value of bandwidth.

The proposed protocol is an efficient routing scheme that improves the efficiency of location-aided routing (LAR). In the proposed scheme, messages can be transmitted in a request zone based on the minimum, maximum slope limit. This limitation will help in reduction in flooding of RREQ packet and in turn helpful in reduction in bandwidth consumption. Bandwidth consumption can be further reduced by using the concept of minimum required bandwidth from the source node to the destination node. Bandwidth is use to check the minimum required bandwidth on every node which receive the RREQ packet and forward the RREQ packet to next node.

Based on the analysis of ELBPAR, proposed protocol improves the QOS in terms of bandwidth and reduces the RREQ flooding. This proposed protocol is use bandwidth factor to provide more efficient path for communication from source to destination mobile node.

"Enhance Location Based Power Aware Routing" is a protocol which helps in dealing with the two major quality factors bandwidth and RREQ flooding. But there is one more important quality factor named as "Cell loss ratio" that also needs to be handled. Also, limited power is another reason for link failure and in turn packet drop. This protocol can further be enhanced on the bases of power enable routing along with RREQ and RREP packet. So, new protocols are required to provide more Enhance paths along with basic QoS factors i.e. bandwidth and delay.

\section{REFERENCES}

[1]. Natrajan Meghanathan, "A Location PredictionBased Reactive Routing Protocol to Minimize the Number of Route Discoveries and Hop Count per Path in Mobile Ad Hoc Networks" Department of Computer Science, Jackson State University, Jackson, MS 39217, USA.

[2]. Y.B. Ko and N. H. Vaidya, "Location-Aided Routing in Mobile Ad Hoc-Scale for Ad Hoc Networks," Proceedings of the second ACM international symposium on Mobile ad hoc networking \& computing, Long Beach, CA, USA, pp. 22-32, October 2001

[3]. G. Dommety and R. Jain. "Potential Networking Applications of Global Positioning System (GPS)," Technical Report TR-24, Computer Science Department, The Ohio State University, April 1999

[4]. M. Mauve, J. Widmer, and H. Hartenstein. A Survey on Position-Based Routing in Mobile Ad-Hoc Networks. IEEE Network, 15(6):30-39, November/December 2001

[5]. D. D. Perkins and H. D. Hughes, "A survey on quality-of-service support for mobile ad hoc networks," Wireless Communications and Mobile Computing, 2002

[6]. . P.K.Suri Dr. M. K. Soni, Mrs. Parul Tomar" Location Based Power Aware Routing" 
[7]. Martin Mauve and Jörg Widmer, "A Survey on Position-Based Routing in Mobile Ad Hoc Networks", IEEE November/December 2001, pp. 31-39

[8]. Wenzhu Zhang, Jiandong Li and Xuan Wang, "CostEfficient QoS Routing Protocol for Mobile Ad Hoc Networks", Proceedings of the 19th International Conference on Advanced Information Networking and Applications (AINA'05).

[9]. Nen-Chung Wang , Si-Ming Wang, "An Efficient Location-Aided Routing Protocol for Mobile Ad Hoc Networks",11th International Conference on Parallel and Distributed Systems (ICPADS'05), 2005

[10].Tzay-Farn Shih , Hsu-Chun Yen, "Location-aware routing protocol with dynamic adaptation of request zone for mobile ad hoc networks", Springer Wireless Netw (2008) 14:321-333,DOI 10.1007/s11276-006-9955-y 